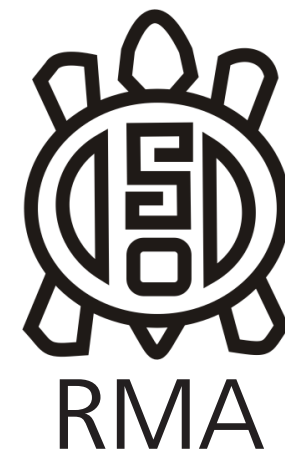

Museología

\title{
Análisis de la colección bioarqueológica perteneciente al Museo Arqueológico de Villa Paranacito (Prov. de Entre Ríos)
}

\author{
Analysis of the bioarchaeological collection belonging to the \\ Archaeological Museum of Villa Paranacito (Prov. of Entre Rios) \\ Mariano C. Del Papa* \\ *División Antropología. Facultad de Ciencias Naturales y Museo. Universidad \\ Nacional de La Plata. Argentina. E-mail: mdelpapa@fcnym.unlp.edu.ar
}

\begin{abstract}
Resumen
El objetivo de este trabajo es presentar los resultados obtenidos en la intervención preventiva y la caracterización de la estructura general de la colección bioarqueológica, bajo la guarda del Museo Arqueológico de Villa Paranacito. La estrategia utilizada, para la efectivización de un abordaje acorde, fue organizada en dos etapas. En la primera, se hizo una evaluación de las condiciones de almacenamiento y la información contextual. En la segunda etapa, se desarrolló un protocolo dirigido a tareas de conservación preventiva, las cuales fueron diseñadas para organizar el repositorio y elaborar una base de datos. De este modo, se logró determinar que las procedencias de los restos óseos corresponden a los sitios arqueológicos El Guazuncho (NMI=1) y Arroyo Martínez (NMI=5). Asimismo, se obtuvo un fechado radiocarbónico en el sitio El Guazuncho. Los resultados obtenidos, posicionan a este sitio arqueológico en el Holoceno tardío final. Finalmente, con este trabajo se aportan datos sobre patologías que permitieron su contextualización regional.
\end{abstract}

Palabras clave: Delta del río Paraná; Museo; Conservación; Bioarqueología.

\begin{abstract}
The aim of this work is to present the results obtained in the preventive intervention and the characterization of the general structure of the bioarchaeological collection, under the care of the Archaeological Museum of Villa Paranacito. The strategy used, for the realization of a suitable approach, was organized in two stages. In the first, an evaluation of storage conditions and contextual information was made. In the second stage, a protocol was developed aimed at preventive conservation tasks, which were designed to organize the repository and create a database. In this way, it was possible to determine that the provenances of the skeletal remains correspond to the archaeological sites El Guazuncho $(\mathrm{NMI}=1)$ and Arroyo Martínez $(\mathrm{NMI}=5)$. Likewise, a radiocarbon dating was obtained at the El Guazuncho site. The obtained results position this archaeological site in the final late Holocene. Finally, this work provides data on pathologies that allowed its regional contextualization.
\end{abstract}

Keywords: Paraná River Delta; Museum; Conservation; Bioarchaeology.

\section{Introducción}

Los estudios sobre las sociedades humanas prehistóricas que han habitado el actual territorio constituido por el delta del río Paraná, han fundamentado sus investigaciones, en primer lugar, a partir de la utilización de materiales arqueológicos y bio arqueológicos frutos de excavaciones sistemáticas como también mediante el uso de colecciones bajo guarda institucional en diferentes museos del país. En los últimos años se ha visto acrecentado el interés por colecciones que se encuentran en museos, que han sido el producto de la acumulación de restos arqueológicos y biarqueológicos a través del tiempo. Los mismos carecían, en muchos casos, de algún tipo de abordaje sistemático debido a que las formas de excavación eran poco o nada documentadas por quienes las obtenían (Castro y Del Papa, 2015). La falta de contextualización de estas colecciones, hace que su potencial como objeto de interés científico sean menor. Sin embargo, un apropiado abordaje preventivo de los materiales constitutivos de las colecciones y la organización de la información disponible, mediante la puesta a punto de una base de datos, permitirá una adecuada visibilización de las mismas.

En la localidad de Villa Paranacito, provincia de Entre Ríos, se encuentra el Museo Regional y Arqueológico de Villa Paranacito. El mismo fue creado como producto de diferentes proyectos pedagógicos - institucionales (cabe consignar que el espacio para su creación fue cedido por el Instituto de Formación Docente Continua de Villa Paranacito), que involucraron a docentes y alumnos de esta institución y que, a través del tiempo, ha creado un importante repositorio de materiales tanto arqueológicos como bioarqueológicos. El objetivo de la creación de este 
espacio fue el de fomentar y enriquecer la discusión sobre la identidad social y cultural de la localidad de Villa Paranacito, poniendo un fuerte énfasis en el pasado prehistórico. En virtud de la ley nacional 25.517, donde se hace explícita la necesidad de arbitrar todas aquellas medidas necesarias para el tratamiento ético de restos humanos que se encuentren bajo la guarda institucional, las autoridades de dicho establecimiento contactaron al personal profesional de la División Antropología del Museo de La Plata con el objetivo de ser asistidos en torno a las problemáticas referidas a la manipulación correcta de estos restos.

Aunque el abordaje en estas colecciones puso luz sobre la existencia de ciertas limitaciones, tanto de índole logístico (accesibilidad limitada a las mismas por distancia o la falta de personal idóneo), como administrativo (la necesidad de gestionar ante autoridades locales y provinciales los permisos necesarios para la intervención), y otros de naturaleza organizacional (ausencia de catálogos o inventariados de los materiales que la componen), la utilización de modernas técnicas y métodos acrecentó el caudal de información y permitió el avance en el conocimiento, estimulando el desarrollo creativo de herramientas metodológicas (Peralta, 2019). En este contexto, es en el que se realizaron una serie de actividades vinculadas al desarrollo de un protocolo curatorial, a partir de diferentes abordajes bioarqueológicos orientados a la contextualización de las colecciones.

Los objetivos de este trabajo son, por un lado, describir el protocolo de intervención preventiva llevado a cabo en la colección osteológica humana bajo la guarda del museo ya mencionado y por otro lado, presentar los resultados obtenidos en los estudios bioarqueológicos de la misma. Esto último permitirá la caracterización en relación al tamaño, integridad, composición sexo-etaria y contextualización cronológica, mediante un fechado radiocarbónico.

\section{Antecedentes}

La localidad de Villa Paranacito se encuentra en el delta Inferior del río Paraná. Las primeras investigaciones arqueológicas, en el área, comienzan entre las últimas décadas del siglo XIX y principios del siglo XX. Desde un primer momento, estos trabajos estuvieron generalmente orientados hacia el estudio de construcciones monticulares de tierra, denominados cerritos o túmulos, que constituyen verdaderas particularidades del paisaje, producto de la actividad antrópica de poblaciones humanas que habitaron esta región durante el Holoceno tardío (Castiñeira Latorre, M., Blasi, A., Bonomo, M., y Apolinaire, E., 2014) y sobre los cuales existen registros en todas las tierras bajas sudamericanas (e.g. Bonomo et al., 2011, Erickson, 2006). En este sentido, encontramos los trabajos pioneros de Estanislao Zeballos y Pedro Pico (1897), en los cuales se lleva a cabo una pormenorizada descripción de las excavaciones realizadas en el Túmulo de Campana. Con el inicio del siglo XX diferentes instituciones nacionales empiezan a comisionar investigadores para llevar a cabo la exploración de diferentes paraderos arqueológicos en el Delta del Paraná. Como resultado de estas primeras aproximaciones se logra la localización, excavación y análisis de varios sitios arqueológicos descriptos como túmulos, como es el caso de Túmulo I Brazo Largo, Túmulo I del Brazo Gutiérrez, Túmulos I y || del Paraná Guazú, Túmulo | Río Carabelas (Torres, 1911), Túmulo II Brazo Largo (Gaggero, 1923), Túmulo A La Glorieta (Castro, 1926), Túmulo del Arroyo Sarandí (Lothrop, 1932), Paraná Ibicuy 1 (Aparicio, 1939), y Túmulo del Arroyo Fredes (Vignati, 1941). Durante la segunda mitad del siglo $\mathrm{XX}$, las investigaciones se orientaron al establecimiento de metodologías sistemáticas (Lafón, 1971) y a la implementación de modelos poblacionales que vincularon al delta del río Paraná con áreas adyacentes, mediante la incorporación de fechados radiocarbónicos. Uno de estos primeros trabajos fue el realizado por la Dra. Amanda Caggiano (1984), con la publicación de cuatro fechados radiocarbónicos, provenientes de los sitios arqueológicos Rodeo Viejo de la Nena (1.420 \pm 80 AP y $1.420 \pm 80$ AP) y Don Santiago $(1300 \pm 90$ y $1090 \pm 90)$ situados en cercanías a la localidad de Villa Paranacito.

A partir de la década de 1990, Acosta y Loponte comienzan a trabajar en el área correspondiente al delta inferior del río Paraná. Sus tareas fueron enfocadas tanto a localidades arqueológicas ya descriptas por otros autores, como a la descripción de nuevos sitios arqueológicos. Uno de los casos es el denominado sitio arqueológico Cerro Lutz (Acosta y Loponte, 2006), localizado en el margen izquierdo del arroyo Martínez; los autores lo vinculan al yacimiento arqueológico El Aserradero, excavado en la década de 1970 por R.C. Lafón (Lafón, 1971). El sitio está constituido por un conjunto de enterratorios asociados a materiales arqueológicos donde se han realizado cuatro fechados radiocarbónicos correspondientes al Holoceno tardío (976 \pm 42 años; $916 \pm 42$ años; $796 \pm 42$ años y $730 \pm 70$ ).

\section{Metodología}

En una primera instancia fue necesario desarrollar un protocolo acorde a las características de la colección a intervenir. La puesta a punto de este conjunto de procedimientos permitió describir, de forma detallada, la totalidad de los abordajes necesarios para una óptima conservación de los mismos. En virtud de tratarse de una colección de restos óseos humanos, se consideraron aquellos marcos regulatorios, desde un punto de vista tanto legal como ético (Del Papa y Pucciarelli, 2015).

La normativa legal, en torno a la regulación de las actividades curatoriales en colecciones de esta naturaleza, se describen fundamentalmente en la ley nacional 25.517 y su decreto reglamentario 701/10, a la cual la provincia de Entre Ríos se encuentra adherida desde el año 2012. En su artículo 2, señala que: "Los restos mencionados 
en el artículo anterior y que no fueren reclamados por sus comunidades podrán seguir a disposición de las instituciones que los albergan, debiendo ser tratados con el respeto y la consideración que se brinda a todos los cadáveres humanos". Por otro lado, dada la naturaleza de las tareas abordadas y en el contexto del marco legal regulatorio, se consideraron los aspectos éticos que tratan sobre los deberes y principios que afectan a esta profesión, prestando especial atención a lo propuesto por el Código Deontológico del ICOM para Museos (2006), la declaración de la Asociación de Antropología Biológica Argentina (AABA) en Relación con la Ética del Estudio de Restos Humanos (2007), y el Código Deontológico para el Estudio, Conservación y Gestión de Restos Humanos del Poblaciones del Pasado (Aranda et al. 2014).

Una vez establecidos los lineamientos generales para abordar una colección de restos bioarqueológicos, se evaluaron las características particulares de la misma. Entre los puntos considerados, se tuvo en cuenta aspectos tales como localización física del área de almacenaje disponible (cabe consignar que el depósito se encontraba en la planta baja del edificio, lindante al río Paranacito, por lo que se podía ver afectados por los desbordes del mismo). Por otro lado, dado que las actividades se realizaron durante dos visitas, se estableció un cronograma de trabajo.

En la primera etapa se efectuó la evaluación de las características del área de almacenamiento, describiéndose las particularidades de la colección y detallando el tipo de materia prima utilizado para la guarda. El conjunto de materiales está constituido por restos óseos humanos, a los que se suman material cerámico, lítico y restos óseos de fauna ${ }^{1}$. Pudo observarse que la totalidad de los materiales estaban almacenados en repisas de madera, caja de madera, caja de cartón y envolturas de papel, materiales propicios para favorecer la actividad de agentes físicos (humedad) y biológicos (hongos y entomofauna) que actúan en detrimento de la preservación de los huesos (Montoya et al., 2002). También, se constató que las unidades anatómicas no se encontraban aisladas unas de otras, por lo que el constante contacto ha favorecido la pérdida de integridad de las mismas. Por otro lado, las condiciones de organización previa a la intervención eran prácticamente nulas, ya que no se pudo confirmar la existencia de un catálogo o inventariado, como así tampoco se observó la presencia de rotulado en las unidades anatómicas. La información referida a aspectos tales como la contextualización de los hallazgos, los procedimientos utilizados para la excavación de los entierros y la localización de los sitios, ha sido obtenida a partir de diferentes fuentes. En primer lugar, cabe destacar que en la totalidad de las bolsas y cajas relevadas

\footnotetext{
1 En virtud de las limitaciones ya mencionadas y dado la necesidad de la intervención de los restos óseos humanos, se optó por dejar a la cerámica, el material lítico y fauna para una etapa posterior en la intervención.
}

han sido halladas etiquetas donde se menciona el lugar de procedencia. Otra fuente de información fue la obtenida mediante la realización de entrevistas con aquellos vecinos de la localidad de Villa Paranacito que estuvieron involucrados en la formación de este museo. De este modo, ha quedado establecido que la totalidad de los materiales fue obtenida, producto de la recolección ocasional o el trabajo realizado por personas interesadas en problemáticas sobre el pasado del hombre, pero que en ninguno de los casos ha intervenido un profesional.

A partir de la recopilación de datos conseguidos en las entrevistas realizadas, se pudo constatar que los restos óseos mencionados provienen de dos sitios arqueológicos denominados Arroyo Martínez y El Guazuncho (Figura 1). Los restos provenientes del sitio arqueológico Arroyo Martínez fueron obtenidos como producto del hallazgo accidental de huesos humanos expuestos a la intemperie, y cuya localización se encontraba en la propiedad de la familia Lutz. Ante la posibilidad de que estos restos óseos provengan del sitio arqueológico conocido como El Aserradero (Lafon, 1971) y que posteriormente fue excavado por Loponte y Acosta (2006) y denominado Cerro Lutz, se les pidió a los informantes que localizaran en un mapa el lugar de los hallazgos. Como resultado de estas entrevistas se pudo precisar que los restos corresponden al sitio El Aserradero - Cerro Lutz.

En referencia al sitio arqueológico El Guazuncho, cabe destacar la existencia de un documento manuscrito, en posesión de las autoridades del Museo. En este informe se relata que, a principios del año 1996, como producto de las actividades de mejoramiento de un camino interno en la estancia El Guazuncho, a unos 15 metros de la margen norte del arroyo Sagastume Grande (departamento de Isla del Ibicuy, provincia de Entre Ríos), quedaron expuestos en superficie restos óseos humanos. Como resultado de este hallazgo, un grupo de aficionados llevó a cabo un

Figura 1. Localización de los sitios arqueológicos: 1- El Guazuncho, 2- Arroyo Martínez.

Figure 1. Location of archaeological sites: 1- El Guazuncho, 2- Arroyo Martínez.

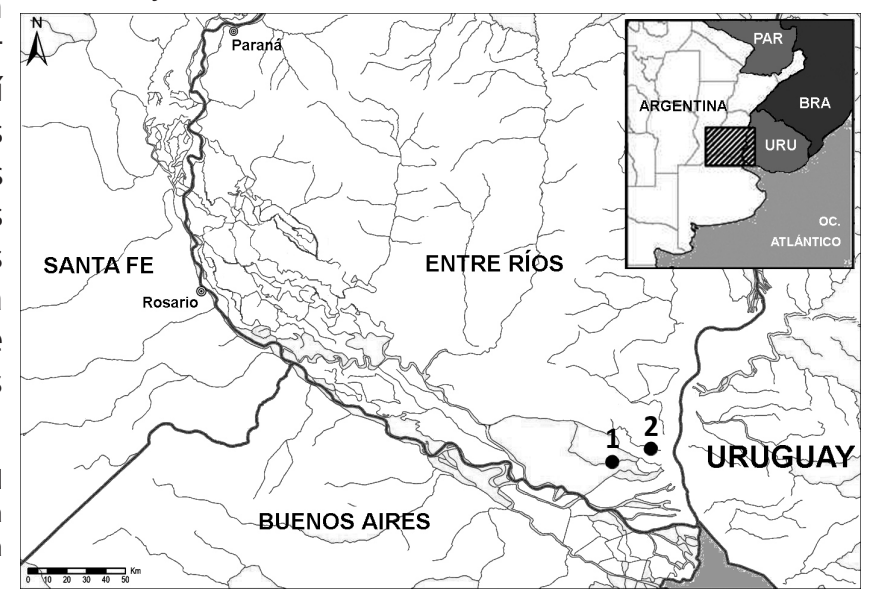


conjunto de tareas, las que consistieron en la extracción de los restos humanos depositados directamente en la matriz sedimentaria y la realización de sondeos sobre el albardón que se encuentra entre el camino y el arroyo Sagastume Grande. Todos estos materiales, junto a la información documental inédita, incluido un esquema sobre el que se describe la disposición del esqueleto, fueron finalmente cedidos por el dueño de la estancia a la colección del Museo Arqueológico de Villa Paranacito. De este modo, se pudo determinar que los restos óseos corresponden a un entierro primario, en posición decúbito ventral extendido, orientado en sentido este - oeste de un individuo (figura 2).

Para la segunda etapa, una vez obtenidos los resultados de la evaluación sobre las condiciones en las que se encontraba la colección y la información disponible, se llevó a cabo el diseño de la base de datos. Su elaboración tuvo como fin obtener una herramienta que permita un óptimo manejo de la colección, restringiendo a la mínima expresión la manipulación de los restos óseos, permitiendo así el menor deterioro posible (Del Papa y Pucciarelli, 2015). En este sentido, fue necesario elaborar un sistema de codificación que permitiera la identificación detallada de las colecciones. Para la confección del mismo, se utilizó una combinación alfanumérica donde quedan expresadas las siglas del nombre del museo (MVP), las siglas del sitio arqueológico de procedencia, Arroyo Martínez (AM) y El Guazuncho (EG), el número de individuo (en caso de poder determinarlo), y número de unidad anatómica. Por otro lado, se consignó toda aquella información de utilidad que permita conocer datos relativos a su localización en el área de almacenaje (número de caja a las que fueron reasignadas cada una de las unidades anatómicas), la conformación de la muestra (número total de individuos, representación etaria y sexual), como así también variables de naturaleza bioantropológica como es el relevamiento de presencia de lesiones patológicas óseas.

Los trabajos curatoriales consistieron, en primer lugar, en separar los restos óseos humanos de los materiales culturales. Cada unidad anatómica fue aislada mediante el uso de bolsas plásticas. A su vez, éstas fueron almacenadas en contenedores plásticos y se reemplazaron así los contenedores de material orgánico (papel, cartón y madera) en los que se encontraban. Se contempló la posibilidad de utilizar técnicas para la limpieza de las unidades anatómicas; sin embargo, dado el grado de descontextualización de los materiales, la pérdida de cualquier evidencia, incluidos los sedimentos asociados, resulte en el desmedro de la contextualización. La totalidad de los materiales que se usaron para el embalaje, almacenamiento y manipulación son libres de ácido, los cuales aseguran la conservación e integridad de los elementos óseos (Del Papa et al. 2010).

Una vez llevado a cabo el reacondicionamiento de la totalidad de los materiales, se comenzó con el inventariado de los elementos óseos y la caracterización de la estructura general de la colección. Como ya ha sido mencionado, las condiciones iniciales para los análisis sobre las características de la colección en estudio, parten de la información obtenida de las diferentes fuentes ya descriptas y del relevamiento de las propiedades intrínsecas del conjunto óseo (integridad, determinación de sexo, determinación de edad, patologías, etc). En el caso del sitio arqueológico Arroyo Martínez, la información no detalla ningún tipo de contexto funerario; por este motivo se optó como estrategia metodológica tratar al conjunto como huesos aislados (ver Ramos van Raap y Bonomo, 2016) y realizar el cálculo de diferentes índices de abundancia como número mínimo de especímenes (NISP), número mínimo de elementos (NME), número mínimo de individuos (MNI)), como así también se calculó el valor de las unidades anatómicas mínimas (MAU) y porcentaje de unidades anatómicas mínimas (MAU\%) (Klein y Cruz Uribe 1984; Lyman, 1984, 1994) con el objetivo de saber cuáles son las partes más representadas del conjunto. En el caso del sitio El Guazuncho, la información disponible permite observar que la disposición de las unidades anatómicas, en la matriz sedimentaria (ver figura 2) no muestra alteraciones, por lo que se determinó que corresponde a un entierro primario. Para conocer la intensidad en el deterioro, se calculó el índice de fragmentación. El mismo fue realizado siguiendo la propuesta de Mondini (2003) utilizando la relación MNE/NISP, cuya variación se encuentra entre 0 y 1 facilitando su lectura ya que mientras más alto sea el valor obtenido menor será la fragmentación del conjunto (Luna et al. 2012). La determinación del sexo y grupo etario de cada individuo se efectuó a partir de las observaciones

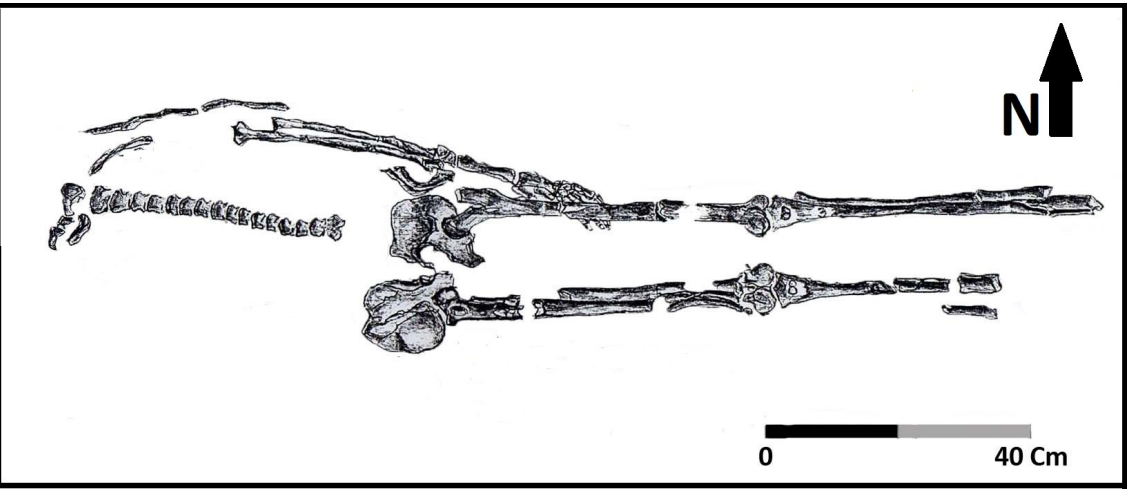

Figura 2. Sitio El Guazuncho, entierro primario decúbito ventral extendido. Tomado y modificado del informe inédito.

Figure 2. El Guazuncho site, extended ventral decubitus primary burial. Taken and modified from the unpublished report 
basadas en distintos rasgos morfológicos diagnósticos de la pelvis (Buikstra y Ubelaker, 1994) y el cráneo (Acsádi y Nemeskéri, 1970 en Buikstra y Ubelaker 1994).

Por otro lado, se realizó el relevamiento paleopatológico. El mismo consistió en el registro de lesiones óseas con etiologías degenerativas (Ortner, 2003, 2012; Brickley e Ives, 2008; Waldron, 2009), y de probable origen hereditario o traumático (Mader y Konzelman, 1979; Dayal, P.K., Shoodhan, K.H., y Dave, C.J. 1983) mediante la observación macroscópica de las unidades anatómicas. Se identificaron las unidades anatómicas comprometidas, su lateralidad y la porción del área afectada, también se realizó un registro fotográfico de las lesiones descriptas.

La contextualización cronológica fue realizada solo para el sitio El Guazuncho, mediante un fechado radiocarbónico sobre restos óseos humanos, en el Laboratorio de Radiocarbono - CIG - La Plata.

\section{Resultados}

\section{Sitio El Guazuncho}

Para el conjunto bioarqueológico procedente del sitio El

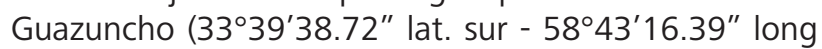
oeste) se establecianalizaron un total de 94 especímenes correspondientes en su totalidad al esqueleto postcraneal. El individuo recuperado tiene una representatividad del $72 \%$ del esqueleto, ya que se hallan ausentes el cráneo, el húmero, cúbito, radio, carpos, metacarpos y falanges de la mano de lateralidad izquierda, costillas, clavículas y escápulas. El estado de preservación de las unidades anatómicas presentes posee un IF de 0.25 , indicando una mala conservación (Mondini, 2003).

Los análisis bioarqueológicos permitieron determinar que este esqueleto correspondería a un individuo masculino según los rasgos observados en la sínfisis púbica y la morfología de la rama superior del pubis (Buikstra y Ubelaker 1992). También se pudo determinar que corresponde al grupo etario adulto.

Para este sitio se cuenta con un fechado radiocarbónico realizado sobre el fémur izquierdo. El fechado obtenido fue de $1220 \pm 60$ años AP (LP-3364; fémur; $\delta^{13} \mathrm{C}=-20 \pm$ 2\%o) (Tabla 1).

El relevamiento macroscópico de la superficie de las unidades anatómicas analizadas permitió reconocer la presencia de lesiones óseas. De este modo, se logró identificar la presencia de labiaciones moderadas marginales (Rogers y Waldron, 1995; López-Barrales et al.
2015), localizadas en la epífisis distal de ambos fémures, con especial énfasis en el fémur izquierdo, como así también en ambas rótulas (figura $3 \mathrm{a}, \mathrm{b}$ ). Por otro lado, se registró la existencia de una osteofitosis (Jurmain y Kilgore 1995) de desarrollo leve en el margen superior anterior de la primera vértebra lumbar $\left(\mathrm{L}_{1}\right)$ (figura $3 \mathrm{c}$ ).

Este tipo de patologías degenerativas son muy frecuentes en el registro bioarqueológico (Ortner, 2003; Waldron, 2012). El estudio sobre su prevalencia y características en poblaciones pre-históricas de cazadores, recolectores y pescadores que habitaron áreas tales como la cuenca inferior del río Uruguay (Ramos Van Raap, 2018; Scabuzzo y Ramos Van Raap, 2019), el delta del río Paraná (Scabuzzo, C., Ramos van Raap, M.A., Bonomo, M., y Politis, G. 2015; Del Papa M.C., Scabuzzo, C., Ramos Van Raap, M.A., Bonilla, D., Pennini, V. 2016) y la costa occidental del río de La Plata (Del Papa, M.C., Scabuzzo, C., Ramos Van Raap M.A., Atencio J.P., Bollini, G., y Bonilla, D. 2020) han tomado particular protagonismo en los últimos años. Estudios paleopatológicos comparativos entre muestras de poblaciones del delta del río Paraná y de la cuenca sur del río Uruguay han demostrado la presencia de lesiones patológicas óseas en diferentes articulaciones, siendo las unidades anatómicas más afectadas, la columna vertebral y las menos afectadas. las rodillas (Scabuzzo y Ramos Van Raap, 2019). A la luz de los resultados obtenidos por estos autores, la tendencia de las lesiones óseas descriptas en este trabajo, podría demostrar una predisposición similar de las poblaciones representadas por los restos óseos estudiados para este sitio arqueológico. Sin embargo, factores tales como el tamaño de la muestra analizada $(n=1)$, y el estado de conservación, tanto del esqueleto axial como apendicular, impide realizar análisis comparativos más riguroso de naturaleza estadísticas.

\section{Sitio Arroyo Martínez}

Para el conjunto bioarqueológico procedente del sitio Arroyo Martínez se analizaron un total de 137 especímenes, de los cuales $10(8,2 \%)$ corresponden al esqueleto craneal y los restantes $112(91,8 \%)$ pertenecen al postcráneo. El estado de conservación de las unidades anatómicas presentes posee un IF de 0.92, indicando una buena preservación de éstas (Mondini, 2003). El cálculo del MAU \% muestra que las unidades anatómicas mejor representadas son la mandíbula y el cráneo (tabla 2 - figura 4). La presencia de estos huesos permitió estimar un $\mathrm{NMI}=5$. Se estableció que los cinco individuos son adultos y se determinó que dos corresponden al sexo masculino, siendo indeterminado el sexo para los restantes individuos (tabla 2).

\begin{tabular}{|c|c|c|c|}
\hline $\begin{array}{c}\text { Sitio } \\
\text { arqueológico }\end{array}$ & Edad AP & $\begin{array}{c}\text { Edad Cal. AD (1 } \boldsymbol{\sigma}) \\
\text { [comienzo: fin / (área relativa] }\end{array}$ & Laboratorio \\
\hline \multirow{2}{*}{ El Guasuncho } & $1220 \pm 60$ & $\begin{array}{l}773: 824 / 0,323351 \\
829: 899 / 0,458067\end{array}$ & LP- 3364 \\
\hline
\end{tabular}

Tabla 1. Fechado radiocarbónico correspondiente al individuo del sitio $\mathrm{El}$ Guazuncho.

Table 1. Radiocarbon dating for the El Guazuncho archaeological site. 


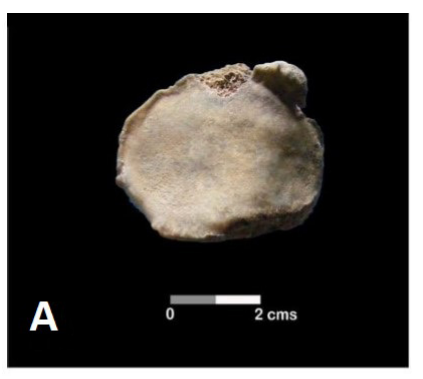

Figura 3. 3a. Rótula derecha con osteofitos, 3b. Fémur derechos con labiaciones, 3c. Vértebra lumbar con desarrollo esteofitos.

Figure 3. 3a. Right patella with osteophytes, 3b. Right femur with labia, 3c. Lumbar vertebra with development of

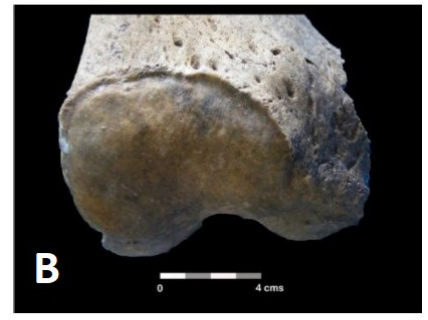
steophytes.

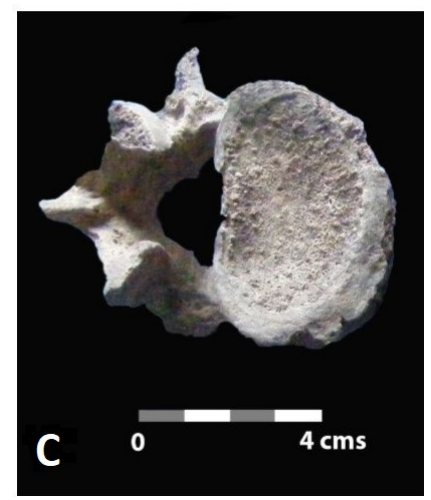

El relevamiento macroscópico de la superficie de las diferentes unidades anatómicas correspondientes a los restos óseos humanos provenientes del sitio Arroyo Martínez, permitió detectar en uno de los cráneos la presencia de transposición en piezas dentales tanto en la arcada dentaria superior, como en la inferior.

En la arcada superior se registra la ausencia, por pérdida post mortem, de ambos terceros molares; el resto de las piezas dentales se encuentran presentes, observándose el proceso de transposición en el desplazamiento del primero y segundo premolar izquierdo (figura 5 a). La arcada dentaria inferior presenta un buen estado de conservación con la totalidad de las piezas dentarias, dando cuenta del proceso de transposición entre el primero y segundo premolar (figura $5 \mathrm{~b}$ ).

La etiología de esta anomalía puede ser entendida como el resultado de la influencia de múltiples factores, tales como la migración de los gérmenes durante la erupción, la herencia genética, dientes deciduos retenidos (Mader y Konzelman, 1979) o eventos de trauma durante la infancia en las edades de 1.5 a 6 años (Dayal, P.K et al. 1983). Otro factor mencionado es la persistencia o retención de dientes deciduos o temporales que puede ocasionar la desviación de la posición normal del diente permanente, dando como resultado la transposición dental (Peck y Peck, 1995; Chattopadhyay y Srinivas, 1996). También se encuentran los factores hereditarios, ligados a desordenes en el ADN que conllevan a la transmisión a los miembros de una misma familia (Peck, S., Peck, L., y Hirsh, G., 1997). En este sentido se ha descripto la presencia en altas frecuencias de transposición del canino y el tercer premolar del maxilar en poblaciones humanas prehistóricas en América del norte, en un contexto de aislamiento geográfico, sugiriéndose que el alto número de casos podría estar vinculado a procesos endogámicos (Nelson, 1992).

Estudios sobre poblaciones actuales, han establecido que la incidencia de este tipo de anomalía es bajo (0,2 \% a 0,38\%) (Thilander y Myrberg, 1973; Yilmaz, H.H., Türkkahraman, H., y Sayin M.O., 2005), registrándose en la arcada maxilar las mayores frecuencias (76\%) entre las cuales el $88 \%$ surgen de manera unilateral (Sherriff y Cobourne, 2006). Las piezas dentarias más afectadas son los caninos (90 $\%)$ a diferencia de los premolares (71\%) y los incisivos laterales (20\%) (Peck y Peck, 1995). También puede estar presente tanto en el maxilar como en la mandíbula (Tseng, Y.C., Chang, H.P., y Chou, T.M.C., 2005).

Este caso presenta particular relevancia debido a la ausencia bibliográfica acerca de registros de transposición para el área correspondiente al delta del río Paraná y sectores fluviales aledaños como cuenca inferior del río Uruguay, cuenca del río Paraná medio y costa occidental del río de La Plata.

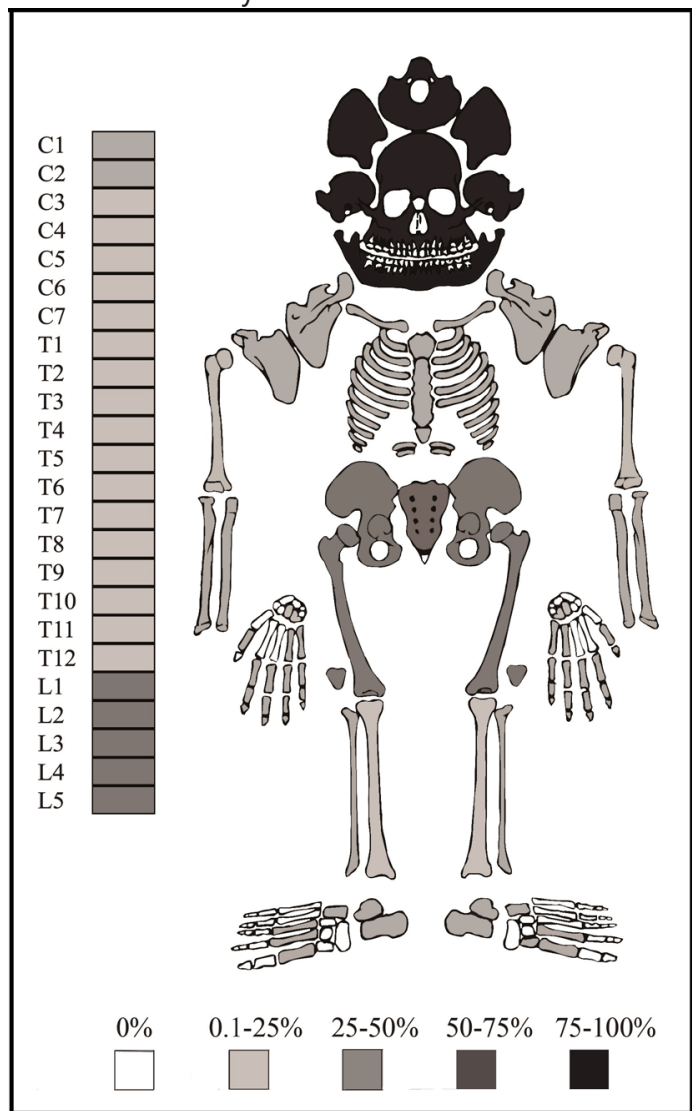

Figura 4. MAU \% correspondiente al conjunto óseo del sitio Arroyo Martínez.

Figure 4. MAU\% corresponding to the bone ensemble of the Arroyo Martínez site. 

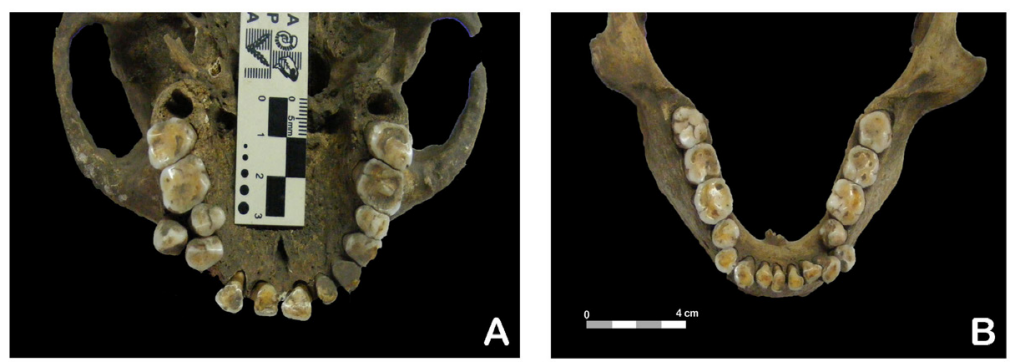

Figura 5. A- Vista oclusal de arcada dentaria superior. B- Vista oclusal de arcada dentaria inferior.

Figure 5. A- Occlusal view of the upper dental arch. B-Occlusal view of the lower dental arch.

por la interrelación de múltiples aristas (discursos) y múltiples líneas de abordaje, que deben ser priorizados según un criterio. El mismo, tomará como directriz aquellos aspectos relacionados, en primera instancia, a la ética de la práctica favoreciendo los reclamos de aquellos sectores sociales que se consideren afectados. Se debe tener en cuenta la existencia de un equilibrio, que permita continuar con los desarrollos científicos, sin que esto vaya en detrimento de la integridad de las colecciones. En su utilización no debe haber sectores de la sociedad que tengan más prioridad que otros, ya que el uso que se haga de ellas no está relacionado al contacto directo con las mismas. Comprender que son parte de nuestro patrimonio e identidad, las vinculan con cada uno de los integrantes de la misma y nos posiciona como participantes activos ante las ellas (Del Papa, M; Vázquez, R; Mune, M.C. y Di Bastiano, A. 2010).

Por esta razón, la intervención en la colección del

En este sentido, será ineludible considerar que las colecciones de esta naturaleza, vistas como objeto de interés para toda la sociedad, deben ser comprendidas (significadas)

\begin{tabular}{|c|c|c|c|c|c|c|c|c|c|c|c|c|c|}
\hline \multirow{3}{*}{ ELEMENTOS } & \multicolumn{13}{|c|}{ Arroyo Martínez } \\
\hline & \multirow{2}{*}{ NISP } & \multicolumn{4}{|c|}{ LF } & \multicolumn{4}{|c|}{$\mathrm{F}$} & \multirow{2}{*}{ NMI } & \multirow{2}{*}{ NME } & \multirow{2}{*}{ MAU } & \multirow{2}{*}{ MAU\% } \\
\hline & & D & $\mathrm{Iz}$ & $A$ & In & D & Iz & $A$ & In & & & & \\
\hline Astrágalo & 2 & - & - & - & - & 1 & 1 & - & - & 1 & 2 & 1 & 20 \\
\hline Atlas & 1 & - & - & - & - & - & - & 1 & - & 1 & 1 & 1 & 20 \\
\hline Axis & 2 & - & - & - & - & - & - & 2 & - & 2 & 2 & 0,5 & 10 \\
\hline Calcáneo & 1 & - & - & - & - & - & 1 & - & - & 1 & 1 & 0,5 & 10 \\
\hline Clavícula & 2 & - & - & - & - & 1 & 1 & - & - & 2 & 2 & 1 & 20 \\
\hline Costilla & 27 & - & - & - & - & 7 & 20 & - & - & 2 & 27 & 1,12 & 22 \\
\hline Cranium & 10 & - & - & - & - & - & - & 4 & - & 4 & 4 & 4 & 80 \\
\hline Cúbito & 1 & - & - & - & - & - & 1 & - & - & 1 & 1 & 0,5 & 10 \\
\hline Cuboide & 2 & - & - & - & - & 1 & 1 & - & - & 1 & 2 & 1 & 20 \\
\hline Cuneiforme 3 & 1 & - & - & - & - & 1 & - & - & - & 1 & 1 & 0,5 & 10 \\
\hline Esternón & 2 & - & - & - & - & - & - & 1 & - & 1 & 1 & 1 & 20 \\
\hline Escápula & 2 & 1 & 1 & - & - & - & - & - & - & 1 & 2 & 1 & 20 \\
\hline Falanges mano & 19 & - & - & - & - & - & - & - & 19 & 1 & 19 & 0,68 & 14 \\
\hline Fémur & 3 & - & - & - & - & 1 & 2 & - & - & 2 & 3 & 1,5 & 30 \\
\hline Ganchoso & 1 & - & - & - & - & - & 1 & - & - & 1 & 1 & 0,5 & 10 \\
\hline Grande & 1 & - & - & - & - & 1 & - & - & - & 1 & 1 & 0,5 & 10 \\
\hline Húmero & 2 & - & - & - & - & 1 & 1 & - & - & 1 & 1 & 0,5 & 10 \\
\hline Mandibula & 6 & - & - & - & - & - & - & 5 & - & 5 & 5 & 5 & 100 \\
\hline Metacarpo & 5 & - & - & - & - & 2 & 2 & - & 1 & 1 & 5 & 0,5 & 10 \\
\hline Metatarso & 5 & - & - & - & - & 3 & 2 & - & - & 1 & 5 & 0,5 & 10 \\
\hline Sacro & 9 & - & - & - & - & 6 & 3 & - & - & 3 & 3 & 3 & 60 \\
\hline Radio & 1 & - & - & - & - & 1 & - & - & - & 1 & 1 & 0,5 & 10 \\
\hline Rótula & 3 & - & - & - & - & 2 & 1 & - & - & 2 & 3 & 1,5 & 30 \\
\hline Coxal & 1 & - & - & - & - & - & - & 1 & - & 1 & 1 & 1 & 20 \\
\hline Tibia & 2 & - & - & - & - & 1 & 1 & - & - & 1 & 2 & 1 & 20 \\
\hline Trapecio & 1 & - & - & - & - & 1 & - & - & - & 1 & 1 & 0,5 & 10 \\
\hline $\begin{array}{l}\text { Vértebra } \\
\text { torácica }\end{array}$ & 14 & - & - & - & - & - & - & 14 & - & 2 & 14 & 1,16 & 24 \\
\hline Vértebra cervical & 4 & - & - & - & - & - & - & 4 & - & 2 & 4 & 0,8 & 16 \\
\hline Vértebra lumbar & 7 & - & - & - & - & - & - & 7 & - & 2 & 7 & 1,4 & 28 \\
\hline TOTAL & 137 & & & & & & & & & 5 & 122 & & \\
\hline
\end{tabular}

Tabla 2. Índices de abundancia correspondiente al conjunto óseo del sitio Arroyo Martínez. Referencias: $\mathrm{L} / \mathrm{F}=$ con línea de fusión; $\mathrm{F}=$ fusionados; $\mathrm{D}=$ Derecho; I = Izquierdo; $\mathrm{A}=\mathrm{Axial} ; \mathrm{In}=$ Indeterminado.

Table 2. Abundance indices corresponding to the bone ensemble of the Arroyo Martínez site. References: $L / F=$ with fusion line; $F=$ merged; $D=$ Right; $I=$ Left; $A=$ Axial; $I n=$ Undetermined. 
Museo Arqueológico de Villa Paranacito pone en evidencia que cualquier injerencia en colecciones de esta naturaleza requiere la aplicación de modernos criterios administrativos que incluyan, la utilización de conceptos y técnicas en conservación preventiva (Guichen, 1995) e inventariado. Estas acciones permitirán, por un lado, el manejo acorde con los lineamientos éticos y legales de los restos humanos y, por otro lado, la puesta en valor de las colecciones mediante un adecuado relevamiento de las mismas (Aranda et al. 2010).

Asimismo, será útil recordar que la existencia de este repositorio no sólo intenta dar cuenta de un conjunto de elementos organizados para una mejor preservación, que permiten cumplimentar con las exigencias de la legislación nacional vigentes, sino que a la vez pretende significar su rol institucional, frente a un colectivo social que cada vez, con mayor asiduidad, se interpela sobre el concepto de identidad social y comienza a buscar en evidencias materiales del pasado prehistórico elementos que pueden ser constitutivos de su propia identidad. Este tipo de enfoque toma especial sentido considerando que el rol de los museos en el siglo XXI, dista mucho de aquellas instituciones decimonónicas que, con una fuerte impronta positivista, han ejercido gran influencia durante todo el siglo XX, principalmente en la esfera de la ciencia, y cuyo principal objetivo era la propalación de conocimientos hacia sujetos pasivos (González-Sanz y Torruella, 2015).

\section{Conclusiones}

Como resultado de la utilización de un abordaje multidisciplinario, mediante la aplicación de técnicas curatoriales, el análisis de variables bioarqueológicas y la contextualización cronológica del sitio arqueológico El Guazuncho, se logró la puesta a punto de un protocolo acorde a la problemática surgida. Así, se consiguió organizar y acondicionar la colección de restos óseos humanos prehistóricos del delta inferior del río Paraná, permitiendo la realización de una base de datos. Los resultados obtenidos permitieron estimar que la procedencia de los restos corresponde a dos sitios arqueológicos, sobre los que se calcularon diferentes índices de abundancia y se estimó para el sitio El Guazuncho un $\mathrm{MNI}=1$ y para el sitio Arroyo Martínez un $\mathrm{MNI}=5$. Se realizó la contextualización cronológica del sitio arqueológico El Guazuncho y se relevaron diferentes patologías en las muestras.

Finalmente, esta información, permitirá visibilizar la existencia de una colección inédita, integrando los datos obtenidos a la bibliografía científica, orientada a los estudios bioarqueológicos en el delta del río Paraná.

La Plata, 20 de abril de 2020

\section{Agradecimientos}

A la Sra. Silvia Mahl vecina de la localidad de Villa Paranacito, a las autoridades del colegio secundario $n^{\circ} 2$ e Instituto de
Formación Docente de Villa Paranacito, profesoras Griselda Peccin y Graciela Velázquez. A mis colegas Dr. Guillermo Lamenza y al Lic. Juan Pablo Atencio (División Antropología - MLP), quienes participaron en los trabajos de campo. Además, quiero agradecer a los evaluadores anónimos que han mejorado el trabajo con sus comentarios y sugerencias.

\section{Bibliografía}

Acosta, A. y Loponte, D. (2006). Informe sobre las investigaciones realizadas en el sitio arqueológico "Cerro Lutz", Provincia de Entre Ríos (Humedal del Paraná Inferior). Secretaría de Cultura de la Nación, Instituto Nacional de Antropología y Pensamiento Latinoamericano, Buenos Aires.

Alt, K.W., Rosing, F.W., Teschler-Nicola, M. (1998). Dental anthropology: fundamentals, limits and prospects. Wien, New York: Springer.

Aparicio de., F. (1939). Las culturas indígenas del Río de la Plata. El Paraná y sus tributarios. En R. Levene. (Ed), Historia de la Nación Argentina, 419-422, Buenos Aires, Argentina: El Ateneo.

Aranda, C. (2009). Primera etapa del proyecto de manejo de colecciones osteológicas en el Área de Antropología Biológica, Museo Etnográfico J. B. Ambrosetti, Buenos Aires, Argentina. IV Foro de Conservación del Patrimonio Cultural. Instituto de Estudios Avanzados, 28-29, Caracas, Venezuela.

Aranda, C., y Ramundo, P. (2010). Conservación preventiva y protección patrimonial de los restos bioarqueológicos del Pukara de La Cueva (Departamento de Humahuaca, Provincia de Jujuy). En J. R. Bárcena \& H. Chiavazza (Eds.), Arqueología Argentina en el Bicentenario de la Revolución de Mayo. XVII Congreso Nacional de Arqueología Argentina 221-226, Mendoza, Argentina: Facultad de Filosofía y Letras, Universidad Nacional de Cuyo.

Aranda, C., Zuccala, K., Avido, D.N., Salvarredi, A., Luna, L. y Gigliotti, V. (2010). Manejo de colecciones osteológicas del Museo Etnográfico J. B. Ambrosetti (FFyLUBA). I Congreso Nacional de Museos Universitarios (La Plata, Argentina, octubre 2010). Red de Museos de la Universidad Nacional de La Plata, La Plata.

Aranda, C., Barrientos, G. y Del Papa, M. (2014). Código deontológico para el estudio, conservación y gestión de restos humanos de poblaciones del pasado. Revista Argentina de Antropología Biológica 16 (2): 111-113.

Asociación de Antropología Biológica Argentina. (2007). Declaración de la Asociación de Antropología Biológica Argentina (AABA Ética del Estudio de Restos Humanos. http://www.fcnym.unlp.edu.ar/aabra.

Bonomo, M., Capdepont, I., y Matarrese, A. (2009). 
Alcances y limitaciones en el estudio de colecciones. Los materiales arqueológicos del Delta del río Paraná depositados en el Museo de La Plata. Revista de Arqueología Sudamericana, 5 (1), 68-101.

Brickley, M. y Ives, R. (2008). The archaeology of metabolic bone disease. Academic Press.

Buikstra, J.E., y Ubelaker, D.H. (1994). Standards for data collection from human skeletal remains. Arkansas Archaeological Survey Research Series n. 44. Arkansas, EE.UU.

Caggiano, M.A. (1984). Prehistoria del NE. Argentino. Sus vinculaciones con la República Oriental del Uruguay y Sur de Brasil. Pesquisas, Antropología 38, 117 págs. Instituto Anchietano de Pesquisas. Río Grande do Sul.

Caggiano, M.A., Flores, O.B., Méndez, M.G., y Salceda, S.A. (1978). Nuevos aportes para el conocimiento antropológico del Delta del Paraná. Relaciones de la Sociedad Argentina Antropología, (12) 155-168.

Castro, A. (1926). Informe sobre expedición al Delta del Paraná, Arroyo La Glorieta. La Plata, Buenos Aires, Argentina: Museo de La Plata.

Castro, J.C., y Del Papa, M.C. (2015). La estructura del registro bioarqueológico del río Uruguay inferior. Análisis de la colección osteológica humana del Museo Arqueológico Manuel Almeida (Gualeguaychú, Entre Ríos). Intersecciones en Antropología (16) 195-205.

Castiñeira Latorre, M., Blasi, A., Bonomo, M., y Apolinaire, E. (2014). Modificación antrópica del paisaje durante el Holoceno tardío: Las construcciones monticulares en el Delta Superior del río Paraná, Argentina. Revista de la Asociación Geológica Argentina. (71) 33-47. de Antropología XII: 155-174

Chattopadhyay, A., y Srinivas, K. (1996). Transposition of teeth and genetic etiology. Angle Orthodontist. (66)147-152.

Dayal, P.K., Shoodhan, K.H., y Dave, C.J. (1983). Transposition of canine with traumatic etiology. Journal of the Indian Dental Association (55) 283-285.

Del Papa, M., Vázquez, R., Mune, M.C. y Di Bastiano, A. (2010). Administración de las colecciones osteológicas en la División Antropología - FCNyM. Actas del I Congreso de Museos Universitarios. La Plata, Buenos Aires, Argentina.

Del Papa, M. C., y Pucciarelli, H.M. (2015). Administración de las colecciones de antropología biológica del Museo de La Plata. Revista Argentina de Antropología Biológica. 17(2).1-5

Del Papa M.C., Scabuzzo, C., Ramos Van Raap, M.A., Bonilla, D., Pennini, V. (2016). Nuevos análisis bioarqueológicos de la colección osteológica del sitio Paraná Ibicuy 1 (Delta inferior del delta del Río Paraná, Entre Ríos). Intersecciones en Antropología (17). 263 - 267. Nota breve.

Del Papa, M.C., Scabuzzo, C., Ramos Van Raap M.A., Atencio J.P., Bollini, G., y Bonilla, D. (2020). Reanálisis de las muestras bioarqueológicas provenientes de los sitios Palo Blanco y Los Talas (partido de Berisso, provincia de Buenos Aires). Intersecciones en Antropología. En prensa.

Ely, N.J., Sherriff, M., y Cobourne M.T. (2006). Dental transposition as a disorder of genetic origin. European Journal of Orthodontics. 28(2)145-151

Erickson, C. (2006). The Domesticated Landscapes of the Bolivian Amazon. Time and Complexity en W. Balée y C. Erickson (Eds.) Historical Ecology: Studies in the Neotropical Lowlands. 235-278. New York, EE.UU.: Columbia University Press.

Gaggero, P. (1923). El túmulo indígena II del Brazo Largo (Delta del Paraná - Provincia de Entre Ríos) explotado en el mes de mayo de 1923. (Preliminar). Universidad Nacional de La Plata, Instituto del Museo. 1924. Nros 6065-78. 7pp. Ms.

Gatto, S. (1939). El paradero-cementerio de Brazo Largo (Delta del Paraná). Physis (16) 365-376.

González-Sanz, M. y Torruella, M. (2015). Educación patrimonial e identidad. El papel de los museos en la generación de cohesión social y de vínculos de pertenencia a una comunidad. CLIO. History and History teaching. 41. 235-252.

Guichen, G. de. (1995). La conservarion préventive: un changement profond dementalité. Cahiers d'étude. ICOMCC. museum/study_series_pdf/1_ICOM.pdf

Jurman, R. D. y Kilgore. L. (1995). Skeletal evidence of osteoarthritis: a palaeopathological perspective. Annals of the Rheumatic Diseases 54: 443-450.

Klein, R.G. y Cruz-Uribe K. (1984). The Analysis of Animal Bones from Archaeological Sites. University of Chicago Press, Chicago.

International Council of Museums (ICOM). (2006). Código Deontológico para Museos. http://archives.icom.museum/ codigo.html (27 de marzo de 2014).

Lafon, C. R. (1971). Introducción a la arqueología del Nordeste argentino. Relaciones de la Sociedad Argentina Antropología (2) 119-152.

Laptook, T., Silling, G. (1983). Canine Transpositionaproaches to treatment. The Journal of the American Dental Association (107) 746-748.

López-Barrales, R., Hubbe, M., Aspillaga, E., Neves, W., Niemeyer, H. (2015). Osteofitosis vertebral en poblaciones prehispánicas de San Pedro de Atacama, norte de Chile. Estudios Atacameños Arqueología y Antropología 
M. C. Del Papa| Revista del Museo de Antropología 13 (3): 165-174 | 2020

DOI: http://doi.org/10.31048/1852.4826.v13.n3.28255

Surandinas. N 50. pp. $177-194$.

Lothrop. S. K. (1932). Indians of the Paraná Delta, Argentina. Annals of the New York Academy of Science (32) 77-232.

Luna, L., C. M. Aranda, S. García Guraieb, T. Kullock, A. Salvarredy, R. Pappalardo, P. Miranda y H. Noriega. (2012). Factores de preservación diferencial de restos óseos humanos contemporáneos de la "Colección Chacarita" (Ciudad Autónoma de Buenos Aires, Argentina). 14: 53-67

Lyman R. (1984). Bone density and differential survivorshipof fossil classes. Journal of Anthrop Arch 3:259-299.

Lyman, R. L. (1994). Vertebrate taphonomy., Cambridge, Inglaterra: Cambridge University Press.

Mader, C. y Konzelman, J.L. (1979). Transoisition of teeth. Journal of the American Dental Association. 98:412-413

Meindl, R.S. y Lovejoy, C.O. (1985). Ectocranial Suture Closure: A Revised Method for the Determination of Skeletal Age at Death Based on the Lateral-Anterior Sutures. American Journal of Physical Anthropology (68) 57-66.

Mondini, M. (2003). Formación del registro arqueofaunístico en abrigos rocosos de la Puna argentina. Tafonomía de carnívoros. (Tesis doctoral). Facultad de Filosofía y Letras. Universidad de Buenos Aires.

Mader, C., y Konzelman, J.L. (1979). Transoisition of teeth. Journal of the American Dental Association (98) 412-413.

Nelson, G.C. (1992). Maxillary canine/third premolar transposition in a prehistoric population from Santa Cruz Island, California. American Journal of Physical Anthropology 88(2)135-44.

Ortner, D. (2003). Identification of Pathological Conditions in Human Skeletal Remains. Nueva York, EE.UU.: Academic Press.

Ortner, D. J. (2012). Differential diagnosis and issues in disease classification. En A. Grauer (Ed.), A companion to paleopathology (pp. 250-267). John Wiley \& Sons.

Peralta, E.A. (2019). Demografía humana, dieta y actividad en los límites de la dispersión agrícola: tendencias bioarqueológicas en el sur de Mendoza a finales del Holoceno tardío. (Tesis doctoral) Facultad de Filosofia y Letras - UBA.

Peck, S., y Peck, L. (1995). Classification of maxillary tooth transpositions. American Journal of Orthodontics and Dentofacial Orthopedics 107(5) 505-517.

Peck, S., Peck, L., y Hirsh, G. (1997). Mandibular lateral incisor - canine transposition in monozygotic twins. Journal of Dentistry for Children (64) 409-413.
Ramos van Raap, M. A. y Bonomo M. (2016). Nuevos estudios de la colección bioarqueológica de los sitios Arroyo Malo, El Cerrillo y Arroyo Sarandí (Delta del Paraná). Intersecciones en Antropología, volumen especial 3: 71-82.

Ramos van Raap, M.A. (2018). Paleopatologia en poblaciones prehispanicas del sur del Noreste Argentino (Tesis Doctoral). Facultad de Ciencias Naturales y Museo, UNLP, La Plata.

Rogers, J. y Waldron, T. (1995). A Field Guide to Joint Disease in Archaeology. West Sussex, Estados Unidos: John Wiley \& Sons

Rostain, S. (2012). Islands in the Rainforest Landscape Management in Pre-Columbian Amazonia. Walnut Creek, California, EE.UU.: Left Coast Press.

Scabuzzo, C., Ramos van Raap, M.A., Bonomo, M., y Politis, G. (2015). Estudios bioarqueologicos en el sitio Los Tres Cerros 1 (Delta Superior del rio Parana, Entre Rios, Argentina). Boletim do Museu Paraense Emilio Goeldi. Ciencias Humanas, 10 (2), 509-535.

Scabuzzo C., y Ramos Van Raap M.A. (2019). Alcances y limitaciones en el relevamiento de osteoartrosis. Primeros resultados de los análisis en series osteológicas del sur de Entre Ríos (Argentina). En Fabra, Novellino, Arrieta y Salega (Eds.). Metodología para el estudio de restos esqueletales en Argentina: Nuevas perspectivas. 97-125. Córdoba, Argentina

Thilander, B., y Myrberg., N. (1973) The prevalence of malocclusion in Swedish schoolchildren. Scandinavian journal of dental research 81(1)12-21.

Torres, L.M. (1903). Los cementerios indígenas del sur de Entre Ríos y su relación con los del Uruguay, túmulos de Campana (Buenos Aires) y Santos (Brasil). Anales del Museo Nacional de Buenos Aires (9) 57-75.

Torres, L.M. (1911). Los primitivos habitantes delta del Paraná, t. IV. La Plata, Buenos Aires.

Tseng, Y.C., Chang, H.P., y Chou, T.M.C. (2005). Canine traspostion. The Kaohsiung Journal of Medical Sciences 21 (10) 441 - 447.

Waldron, T. (2012). Joint disease. En A. Grauer (Ed.) A Companion to Paleopathology (513-530). Chichester: Wiley- Blackwell.

Yilmaz, H.H., Türkkahraman, H., y Sayin M.O. (2005). Prevalence of tooth transpositions and associated dental anomalies in a Turkish population. Dentomaxillofac Radiology 34 (1) 32-35.

Zeballos, E., y Pico, P. (1878). Informe sobre el túmulo prehistórico de Campana. Anales de la Sociedad Científica Argentina. 6:244-260. 